\title{
Ontologías abundantes y rigidez para expresiones predicativas ${ }^{*}$ \\ (Abundant Ontologies and Rigidity for Predicative Expressions) \\ Ezequiel ZERBUDIS
}

Recibido: 13.09.2012

Versión final: 04.10.2012

BIBLID [0495-4548 (2014) 29: 79; pp. 113-132]

DOI: $10.1387 /$ theoria. 6502

RESUMEN: En este trabajo, critico una estrategia argumental que ha sido utilizada para defender la concepción de la rigidez para expresiones predicativas entendida como identidad de lo designado en los distintos mundos posibles. Se trata de una estrategia basada en la suposición de que las descripciones tendrían la capacidad de designar individuos inusuales ("abundantes") en la misma medida en que ciertas expresiones predicativas (supuestamente) descriptivas serían capaces de designar propiedades inusuales. Señalo que esta suposición no es compatible con ciertos principios que gobiernan el funcionamiento semántico de las descripciones y con la verdad intuitiva de ciertas predicaciones que las incluyen.

Palabras clave: designación; expresión predicativa; rigidez; ontología.

ABSTRACT: In this paper, I criticize an argumentative strategy that has been used in the literature to defend the view according to which rigidity for predicative expressions should be understood as identity of designation across possible worlds. It is a strategy that is based on the supposition that definite descriptions could designate unusual (i.e., "abundant") individuals just as well as some (allegedly) descriptive predicative expressions are able to designate unusual properties. I point out that this presupposition is at odds with some principles governing the semantic behaviour of definite descriptions and with the intuitive truth of some predications that include them.

Keywords: designation; predicative expression; rigidity; ontology.

\section{Introducción}

En este trabajo discuto y critico un presupuesto que está a la base de ciertos argumentos utilizados para defender la tesis según la cual la noción de rigidez para expresiones predicativas debe ser entendida en términos de la identidad de lo designado en los distintos mundos posibles. ${ }^{1}$ Se trata de un presupuesto que concierne, en última instan-

* Querría agradecer a Dan López de Sa y a Gonzalo Rodriguez-Pereyra por sus comentarios a versiones previas de este trabajo.

${ }^{1}$ En el presente trabajo, utilizaré "expresión predicativa" como un término amplio que incluye cualquier tipo de expresión cuya función principal consista en adscribir una propiedad (característica, etc.) a objetos-típicamente, a los objetos designados por ciertos términos singulares con los que se encuentra concatenada. Así, tanto los términos generales como los predicados serán considerados aquí como expresiones predicativas (lo que no implica que me comprometa con que haya una única noción de adscripción que sirva para caracterizar el funcionamiento de estos dos tipos de expresiones, ni tampoco con la tesis contraria; simplemente quiero usar una noción lo suficientemente amplia como para, en primer lugar, poner entre paréntesis la discusión acerca de cuál de estas categorías incluiría las expresiones predicativas primarias, y también, en segundo lugar, acomodar el hecho de que distintos autores que han intervenido en estas discusiones han formulado sus posiciones en relación con una u otra de estas categorías). 
cia, a los tipos de entidad que sería posible denotar mediante ciertas descripciones; en particular, lo que está en juego es la posibilidad que tendrían (o no) ciertas descripciones definidas de designar algunas entidades inusuales hechas a medida para funcionar como sus referentes rígidos. Antes de presentar con mayor detalle este presupuesto y algunas estrategias argumentativas basadas en él resultará conveniente introducir en primer lugar el problema general en el contexto de cuya discusión éstas se inscriben y las líneas generales de las propuestas que se han hecho para resolverlo; una vez hecho esto me ocuparé de examinar con algún detalle dos variantes del tipo de argumentación basado en el presupuesto en cuestión, a saber, una presentada por Joseph LaPorte (2000), y otra que difiere de ésta en ciertos puntos importantes defendida más recientemente por Dan López de Sa (2008); finalmente, en el resto del trabajo, intentaré mostrar por qué la suposición que está a la base de este tipo de argumentación es incorrecta, así como algunas consecuencias generales que pueden extraerse de dicha circunstancia.

\section{La problemática general en la que se inscribe la argumentación}

Comencemos por la primera de estas cuestiones. La problemática más general en la que se inscribe la argumentación que vamos a analizar es la de cómo caracterizar de modo preciso la tesis, formulada por Kripke en la tercera conferencia de El nombrar y la necesidad, según la cual ciertos términos de género natural serían rígidos. Un primer escollo para la clarificación de esta tesis lo encontramos en el hecho de que los términos de género natural no parecen pertenecer, desde un punto de vista formal, a una única categoría de expresiones: así, pareciera que no sólo ciertos términos singulares, tales como 'el agua' o 'la abeja', sino también ciertas expresiones predicativas, tales como '... es agua' '.. es un tigre', o simplemente 'tigre' (entendida como un término general), podrían ser consideradas como términos de género natural—dado que son términos que se relacionan semánticamente, de un modo u otro, con géneros naturales. En todo caso, asumiendo en principio que la noción de rigidez está suficientemente bien definida para los términos singulares, y que, por otra parte, una gran cantidad de términos de género natural sólo podrían ser considerados como expresiones predicativas, el punto crítico para clarificar la tesis de Kripke mencionada más arriba pareciera consistir en ofrecer una explicación de la noción de rigidez que (también) pueda utilizarse para caracterizar a tales expresiones.

En lo que respecta a esta última cuestión, las distintas propuestas positivas formuladas pueden dividirse en dos grandes grupos, según cuál de las relaciones semánticas en las que están involucradas las expresiones predicativas sea tomada en cuenta para determinar su rigidez. En efecto, debe seleccionarse una de tales relaciones como la noción relevante de designación, ya que, como se sabe, Kripke no define una noción de rigidez simpliciter, sino más bien la noción de designador rígido, como queda claro en el pasaje en el que se introduce por primera vez esta noción:

Llamemos a algo un designador rígido si designa al mismo objeto en todo mundo posible, y [llamémoslo] un designador no rígido o accidental si esto no es el caso. (Kripke 1980, 48)

Para comprender el carácter problemático de la extensión de la noción de rigidez a las expresiones predicativas, resulta conveniente tomar en cuenta que, al menos a pri- 
mera vista, hay dos relaciones semánticas distintas en que dichas expresiones se encuentran que podrían ser consideradas como otras tantas relaciones de designación en función de las cuales definir una noción de rigidez. Estas relaciones son las siguientes:

(a) En primer lugar, la relación (que podríamos llamar de "aplicación") en que una expresión predicativa se encuentra con aquellos objetos a los que se aplica con verdad.

(b) En segundo lugar, la relación (que podríamos llamar de "expresión”) en que un término se encuentra con la propiedad que expresa, propiedad que es, a su vez, aquello que se adscribe a ciertos objetos cuando se les aplica el término.

Los dos grupos principales de propuestas que se han formulado respecto de cómo extender la noción de rigidez a expresiones predicativas pueden entenderse como el efecto de tomar a una u otra de estas relaciones como la noción de designación relevante para definir una noción adecuada de rigidez (en caso de que el comportamiento del término en cuestión manifieste cierta constancia, respecto de esa relación, a lo largo de la dimensión modal). Así, correspondiendo a las relaciones señaladas más arriba como (a) y (b), pueden definirse las siguientes nociones de rigidez para expresiones predicativas:

(A) La así llamada noción esencialista de rigidez, según la cual una expresión predicativa será rígida si y sólo si, si se aplica a un objeto en un mundo posible, se aplicará a ese mismo objeto en todo mundo posible (en que el objeto exista). ${ }^{2}$

(B) La así llamada noción de rigidez como identidad de designación, según la cual una expresión predicativa será rígida si y sólo si designa a (i.e., expresa, i.e., es usada para adscribir) la misma propiedad en todo mundo posible.

Como ya habíamos señalado, cuando se lo utiliza en el contexto de esta discusión (la de cómo entender la rigidez para expresiones predicativas), el tipo de argumento que nos interesa examinar es utilizado en ciertos intentos de defender propuestas correspondientes al grupo (B) (como veremos, también se hace uso de él en otras discusiones emparentadas); en particular, se trata de una estrategia utilizada para defender a este tipo de propuestas de la objeción según la cual la noción de rigidez así definida sería trivial, debido a que haría rígidas a todas las expresiones predicativas. La objeción parece prima facie atendible, ya que parece razonable suponer que, dado cualquier predicado de la forma 'es (un) F' (o cualquier término general ' $F$ '), siempre podría suponerse que existe una propiedad, la de ser (un) $F$, que sería designada rígidamente por tal expresión, de lo que se seguiría que toda expresión predicativa resultaría rígida (al menos, esto sería así si asumimos una noción suficientemente "abundante" de propiedad, que garantice la existencia de todas las propiedades

\footnotetext{
${ }^{2}$ Esta condición entre paréntesis se aplica sólo para algunas versiones ("persistentes") de la concepción; en las versiones "obstinadas" esta condición deja de ser relevante (cf. Gómez-Torrente 2006 para la distinción entre estas diversas variantes de la noción).
} 
necesarias). ${ }^{3}$ Pero, por otra parte, parece razonable suponer que una condición mínima de adecuación que debería cumplir cualquier propuesta de análisis de la noción de rigidez para expresiones predicativas es la de que ésta no resulte trivial. La tensión entre estas dos circunstancias explica que quienes favorecen este tipo de propuesta se hayan preocupado, principalmente, por mostrar que su noción preferida de rigidez no es trivial, lo que tratan de hacer, típicamente, mostrando que hay contraejemplos que pueden esgrimirse frente a esta acusación; esto es, mostrando que hay ciertos casos de expresiones predicativas que cabe considerar como no rígidas. Es en el contexto de intentos de esta naturaleza que se presenta el tipo de consideraciones que vamos a analizar en lo que sigue.

\section{Las argumentaciones a analizar}

Pasamos ahora entonces a considerar con cierto detalle un tipo particular de argumentación que ha sido utilizado para defender ciertas concepciones de la rigidez para términos de género natural de la objeción de trivialización. Se trata de una estrategia que, con distintos énfasis y en el contexto de propuestas más o menos divergentes, aparece en trabajos de Bernard Linsky (1984, especialmente 268-9; cf. también su 2006), Joseph LaPorte (2000, en especial 299-301) y Dan López de Sa (2008). Si bien estas distintas propuestas tienen varios aspectos en común, hay que señalar desde un principio que se dan también entre ellas algunas divergencias importantes, en particular en lo que respecta al modo en que cada una de ellas clasifica a los términos de género natural desde un punto de vista formal. Así, mientras que Linsky considera a los términos de género natural fundamentalmente como términos generales (aunque usa a veces también la expresión 'predicados' en relación a ellos) y López de Sa considera que hay que incluir en dicha clase tanto a predicados como a ciertas nominalizaciones (i.e., designadores singulares de géneros) que se pueden formar a partir de ellos de un modo regular, LaPorte concibe a sus "designadores de género" (kind designators) como términos singulares (de un modo un tanto velado en 2000, de modo explícito en 2006).4 Siendo esto así, las consideraciones de LaPorte

\footnotetext{
${ }^{3}$ Según Lewis $(1986,59-69)$ una concepción abundante de las propiedades admite como tales a todas aquellas que pudieran ser representadas por alguna función de mundos posibles a conjuntos de individuos, por poco natural que tal función pueda en principio parecer-por oposición a las propiedades "escasas", que reflejarían ciertas similitudes cualitativas de los objetos que las instancian; nótese que propiedades como la de ser del color del cielo, que discutiremos más adelante, pertenecerían de todos modos a una subclase bastante natural dentro de la clase de las propiedades abundantes así delimitadas. Es importante también notar que en la discusión que analizaremos se presupone una concepción abundante de las propiedades, por ser la que menos favorece a quienes pretenden rechazar la objeción de trivialización, para que el éxito de su respuesta no dependa de la aceptación de presuposiciones metafísicas más favorables pero controvertidas (como ocurriría si se aceptaran sólo universales o propiedades escasas, sobre cuya base se podría ofrecer lo que López de Sa denomina una "solución escasa" al problema, que evitaría la trivialización debido a la inexistencia de algunas de las propiedades relevantes).

${ }^{4}$ Una formulación explicita de esta opción de LaPorte la encontramos en el siguiente pasaje: "Nótese que, (...), tanto aquí como en mi trabajo anterior [i.e., 2000], intento desarrollar una teoría sobre [I at-
} 
parecerían quedar, en sentido estricto, fuera del ámbito de discusión del presente trabajo, cuyo objetivo es el de analizar algunos problemas en relación con la noción de rigidez para expresiones predicativas. Sin embargo, dado que la argumentación desplegada por LaPorte tiene analogías importantes con las de los demás autores, en parte originadas en que la relación entre un término singular para géneros y el género que éste designa es estructuralmente análoga a la que se da entre una expresión predicativa y la propiedad que ella expresa, su examen resultará esclarecedor en este contexto tanto porque ofrece un contraste interesante a la propuesta de López de Sa como porque explicita de modo mucho más desarrollado una concepción tan similar desde un punto de vista formal a la de Linsky (quien, como vimos, la formula para términos generales) que convendrá atender sobre todo a su formulación del argumento. ${ }^{5}$

Comencemos, en todo caso, exponiendo la posición de López de Sa. De un modo general, la estrategia mediante la cual este autor pretende mostrar que algunas expresiones predicativas serían no rígidas (o "flexibles", como él las llama) se basa en la supuesta constatación de que algunas identificaciones entre nominalizaciones canónicas de predicados, esto es, entre expresiones de la forma 'ser (un) F', son verdaderas sólo de modo contingente. ${ }^{6}$ De la verdad de tales identificaciones se seguiría que las nominalizaciones involucradas en cada una de ellas serían designadores de la misma propiedad, mientras que del hecho de que dichas identidades sean verdaderas sólo de modo contingente se seguiría que al menos uno de los términos involucrados sería no rígido. Finalmente, dado que resulta razonable suponer, además, que una nominalización de la forma 'ser (un) F' designa a una propiedad si y sólo si el correspondiente predicado ('es (un) F') la adscribe, se seguiría de lo anterior también el carácter no rígido de los correspondientes predicados-esto es, se seguiría que un mismo predicado podría usarse para adscribir distintas propiedades en distintos mundos posibles, que es lo que se quería demostrar.

Así, por ejemplo, (1) y (2) serían enunciados de identidad del tipo relevante:

(1) Correr es ejercitarse de la manera preferida por José.

(2) Ser azul es ser del color del cielo.

tempt to elaborate on] la rigidez de los términos singulares para propiedades, no de los términos generales" (LaPorte 2006, 330).

${ }^{5}$ Algunos de los presupuestos que voy a criticar cumplen también un papel en trabajos recientes de G. Martí y J. Martínez, quienes, si bien no formulan ninguna versión del argumento que analizaremos en lo que sigue, utilizan un marco conceptual fundamentalmente inspirado en Linsky (cf. en particular Martí y Martínez 2010, 2011; nótese además que, en estos trabajos, sus autores citan a LaPorte, incorrectamente según se desprende de lo que vengo de señalar, como proponiendo una noción de rigidez entendida como identidad de designación "para términos generales").

${ }^{6}$ La definición original "oficial” que López de Sa da de la noción de nominalización canónica está formulada para el caso del inglés, y sostiene que la nominalización canónica de un predicado ha de obtenerse mediante la sustitución de "el primer verbo que contiene [el predicado] por su forma en gerundio" (2008, 3a). Una primera enmienda que debería hacerse a esta definición consiste en que, en castellano, el gerundio inglés debe reemplazarse mediante la forma verbal en infinitivo; una segunda consistiría, creo yo, en reemplazar 'primer verbo' por 'el o los verbos principales' (para dar cuenta de los casos de predicados complejos o de aquellos que involucran usos de auxiliares; como me hace notar un evaluador anónimo, López de Sa es consciente en buena medida de estas limitaciones; cf 2008, n. 6). 
Nuestro autor sostiene entonces, como venimos de decir, que estas dos identidades que involucran nominalizaciones canónicas son, intuitivamente, verdaderas de modo contingente (ya que, cabe suponer, José podría haber preferido otro modo de ejercitarse y, por otra parte, el cielo podría haber sido de otro color que azul). Dado que, por otra parte, 'correr' y 'ser azul' son, según parece, términos rígidos que designan, respectivamente, las propiedades de correr y de ser azul en todos los mundos posibles, pareciera que, dada la verdad de las identidades en las que aparecen, 'ejercitarse de la manera preferida por José' y 'ser del color de cielo' también deberían considerarse como designando, respectivamente, esas mismas propiedades en el mundo actual, aunque, en virtud del carácter contingente de la identidad, es de suponer que designan otras propiedades respecto de otros mundos posibles (en lugar de designar rígidamente, como supone que lo hacen quien sostiene el carácter trivial de la noción de rigidez en cuestión, respectivamente, las propiedades de ejercitarse de la manera preferida por José y de ser del color de cielo).

Si bien esta estrategia argumentativa general desarrollada por nuestro autor tiene, en mi opinión, varios aspectos muy dudosos, comenzando por las intuiciones que López de Sa supone que tenemos respecto de (1) y (2), no me ocuparé de argumentar aquí de modo directo en contra de tales aspectos de su posición (diremos igual algo al respecto hacia el final del trabajo), sino que me centraré en principio en el análisis de una consideración presentada en este contexto para defender y apuntalar la argumentación que venimos de reseñar-quizás debido a que, según podemos presumir, nuestro autor anticipa ya cierta incredulidad de parte de sus lectores. Se trata de una argumentación subsidiaria que se basa de modo crucial en la suposición de que los términos singulares y las expresiones predicativas tienen un comportamiento completamente paralelo en lo que respecta a la influencia que podría tener la aceptación de ciertas tesis ontológicas en la determinación de sus posibles referencias. A partir de esta suposición nuestro autor argumenta luego que, si se supone que tal comportamiento podría ocasionar un problema de trivialización para la noción de rigidez tal como ésta es aplicada a expresiones predicativas, entonces también debería ocasionarlo para la noción de rigidez aplicable a términos singulares; para concluir que, dado que se supone que hay buenas razones para rechazar el carácter trivial de la noción de rigidez para términos singulares, razones estrictamente paralelas podrían utilizarse para rechazar la objeción de trivialización para el caso de las expresiones predicativas.

Analicemos un poco más detenidamente esta argumentación, para poder eventualmente desentrañar y evaluar las presuposiciones sobre las que descansa. Consideremos en particular, para tal fin, el enunciado (2). Nuestro autor sospecha que algunos hablantes podrían manifestar cierta resistencia a aceptar que este enunciado es verdadero de modo contingente, y supone que el rechazo de esa evaluación ( $\mathrm{y}$, de modo general, de la tesis de que ciertas nominalizaciones son no rígidas) podría depender de la suposición de que, si tomamos una actitud suficientemente liberal respecto de las propiedades que estamos dispuestos a aceptar como posibles designata de (nominalizaciones de) predicados, de tal modo que admitamos como existentes, por ejemplo, tanto la propiedad de ser del color del cielo como la de ser azul, podría parecer inevitable suponer que 'ser del color del cielo' designa (rígidamente) a la primera de éstas, mientras que 
sólo 'ser azul', de entre estas dos, designaría (también rígidamente) a la segunda - en cuyo caso, por supuesto, (2) resultaría (necesariamente) falso (y, en todo caso, más allá de si tales suposiciones están realmente o no a la base de las intuiciones de los hablantes, tesis semejantes podrían ser utilizadas para justificar ese tipo de posición). Esto es: nuestro autor conjetura que un motivo que podría utilizarse para justificar una evaluación de ese tipo, y que podría incluso interferir en las intuiciones de muchos hablantes respecto de los enunciados mencionados, dándolos como falsos en vez de verdaderos, es quizás la suposición de que la aceptación de una ontología "abundante" respecto de las propiedades tendría inevitablemente consecuencias para la semántica de sus designadores. ${ }^{7}$

Nuestro autor intentará, por su parte, rechazar la validez de este tipo de consideración, defendiendo la tesis de que la influencia que podría tener la aceptación de propiedades (más o menos) escasas o abundantes sobre la semántica de las expresiones predicativas es similar (o, más aún, estrictamente paralela) a la influencia que tendría la adopción de objetos abundantes o escasos respecto de la semántica de los términos singulares. Sobre esta base, nuestro autor construye un argumento cuya forma podría explicitarse del siguiente modo:

(i) La influencia que tendría la postulación de una ontología abundante o escasa respecto de la aceptación del carácter rígido o no rígido de ciertos términos es prima facie similar para los casos de los términos singulares y de las expresiones predicativas.

(ii) Si esas relaciones entre ontología aceptada y rigidez pueden dar lugar a un problema de trivialización respecto de la rigidez de las expresiones predicativas, también deben dar lugar a un problema similar respecto de los términos singulares.

(iii) No hay problema de trivialización para la noción de rigidez aplicada a términos singulares.

(iv) No hay problema de trivialización para la noción de rigidez aplicada a expresiones predicativas.

El elemento más problemático de este argumento está, en mi opinión, en la tesis expresada mediante la premisa (i). López de Sa intenta convencernos de la plausibilidad de (i) mediante una comparación entre (2) y (3):

${ }^{7}$ Vale la pena dejar en claro que López de Sa no pretende construir un argumento directo contra la trivialización a partir de la aceptación de ontologías abundantes o escasas para los referentes de distintos tipos de expresiones, sino que simplemente pretende que tales consideraciones "motiven" su solución al problema de la trivialización. El argumento que le adscribo puede entenderse entonces como detallando la forma específica en que tendría lugar esa motivación, aunque éste no aparezca como tal de manera explícita en su trabajo. Si bien esta presentación de su postura podría quizás resultar un tanto infiel a su texto, las objeciones que planteo son de todos modos relevantes para evaluar su posición, ya que atacan la idea misma, presupuesta de un modo u otro en su trabajo, de que habría un paralelismo entre los casos de los términos singulares y los predicados, idea que aparece con claridad, por ejemplo, en el siguiente pasaje: "Pero ahora [i.e., una vez que hemos admitido objetos abundantes] surge un problema, paralelo al problema de la trivialización para los predicados, para la flexibilidad (intuitiva) del término singular "el papa"' (López de Sa 2008, 6a). 
(2) Ser azul es ser [d]el color del cielo.

(3) El papa es Joseph Ratzinger.

Lo que nuestro autor quiere defender es que 'ser del color del cielo' puede, en (2), ser tomada como refiriendo a distintos colores en distintas circunstancias, de un modo completamente similar a la manera en que, intuitivamente, una descripción como 'el papa' en (3) es considerada como designando, en los distintos mundos posibles (y tiempos), a personas diversas — en virtud de lo cual dicha expresión sería no rígida (e inconstante). Ahora bien, quien acusa a una propuesta como la de nuestro autor de trivializar la noción de rigidez sostendría, por el contrario, que 'ser del color del cielo' designa más bien a la propiedad de ser del color del cielo; y, en particular, que no podría no designarla, si se presupone que tal propiedad existe, lo que implicaría la existencia de un contraste entre los casos (2) y (3).

Frente a esta línea argumentativa, López de Sa pretende negar que haya efectivamente semejante diferencia entre estos dos casos, sugiriendo que el supuesto contraste que se quiere ver aquí pareciera depender de la aceptación implícita, de un modo que en principio podría parecer injustificado, de presuposiciones ontológicas distintas en esos dos casos: en efecto, podemos considerar que, mientras que el objetor pareciera admitir, por una parte, una ontología de propiedades abundantes, parece al mismo tiempo presuponer, por el contrario, una ontología de individuos escasos. Pero, continúa nuestro autor, si aceptáramos una ontología similar en ambos casos-en particular, si presupusiéramos una ontología abundante de propiedades y admitiéramos también, en consonancia con esto, una ontología abundante de individuos, de acuerdo con la cual contase como un individuo genuino cualquier combinación mereológica (irrestricta) de (partes de) individuos corrientes (o, alternativamente, individuos generados por relaciones de constitución correspondientes), las condiciones para que surja un problema de trivialización de la rigidez tendría lugar tanto respecto de las expresiones predicativas como respecto de los términos singulares (este es, como vimos, el contenido de la premisa (ii)).

Para aclarar el contenido de la tesis defendida aquí por López de Sa, consideremos un ejemplo de objeto abundante que podría resultar relevante en una evaluación del enunciado (3). Dijimos que, en una ontología de particulares abundantes, el resultado de cualquier combinación de operaciones mereológicas (o de relaciones de constitución durante intervalos de tiempo) a partir de objetos ordinarios (que en este contexto podríamos denominar 'objetos escasos' o 'naturales': personas, autos, árboles, etc.) resultará ser un individuo. Podemos definir, entonces, una categoría particular de individuos abundantes, que podemos denominar, siguiendo a Sidelle (1992), 'personas-decargo' (office persons): una persona-de-cargo es un individuo que está constituido, durante distintos intervalos de tiempo y en los distintos mundos posibles, por los diversos individuos escasos (en este caso, personas) que desempeñan un cargo particular en esos mundos y tiempos- $\mathrm{O}$, alternativamente, sustituyendo el lenguaje endurantista por uno perdurantista, se trata de una entidad conformada por partes temporales que coinciden con las partes temporales de distintos objetos usuales (personas) que corresponden a los intervalos (actuales o contrafácticos) durante los que éstos desem- 
peñan ese cargo. ${ }^{8}$ Un ejemplo que da Sidelle, y retoma López de Sa, es el caso de Elpresidente-de-los-Estados-Unidos (denominado 'Prez' por Sidelle), un individuo que está constituido en la actualidad por Barack Obama, estuvo constituido en el 2008 por G. W. Bush, diez años antes por Bill Clinton, y está constituido por John McCain en el presente de algún mundo contrafáctico apropiado, en el que este último hubiera ganado las elecciones de 2008.

Lo interesante de estas entidades en el contexto de nuestra argumentación consiste en que, según suponen Sidelle y López de Sa, ellas aparecen como buenos candidatos (e, incluso, como los candidatos más naturales) para funcionar como los referentes rígidos de expresiones tales como 'el presidente de los Estados Unidos', 'el presidente de Argentina' y 'el papa', de una manera, además, de acuerdo con la cual los enunciados que contienen tales expresiones resultarían tener los mismos valores de verdad, respecto de las distintas circunstancias de evaluación, que tendrían si consideráramos que esas mismas expresiones designan, por el contrario, en cada situación, de manera no rígida, a los distintos individuos ordinarios que cumplen el cargo en cuestión en dicha circunstancia.

Ahora bien, el punto sobre el que quiere llamar la atención López de Sa es que nuestra evaluación de (3) como una verdad contingente no se ve alterada por la suposición de que existe, junto a individuos usuales como Karol Wojtila y Joseph Ratzinger, el individuo "inusual" El-jefe-de-la-Iglesia-Católica. En efecto, aún cuando supusiéramos que este individuo está disponible para ser designado rígidamente por 'el papa', seguimos suponiendo que (3) es una verdad contingente, y evaluamos que esto sería así porque 'el papa' podría haber designado, en una circunstancia contrafáctica (y temporal) adecuada, a una persona distinta de Joseph Ratzinger. Como dice el mismo López de Sa, de la verdad de un enunciado como (3) se sigue que,

[...] dado que '[Joseph] Ratzinger' no designa [does not signify] El-jefe-de-la-Iglesia-Católica (con respecto al mundo actual), tampoco lo hace 'el papa'. En consecuencia, aún si existe un objeto tal como El-jefe-de-la-Iglesia-Católica, contamos con las razones requeridas para sostener que 'el papa' no lo designa rígidamente $(2008,6 \mathrm{~b}) .{ }^{9}$

8 Agradezco a un evaluador anónimo por obligarme a precisar mejor la estructura ontológica de las personas-de-cargo, en particular respecto de cómo explicitarla en marcos tri- o tetradimensionalistas. En todo caso, y con independencia de cómo se resuelva la cuestión de si los dos marcos son o no, en general, equivalentes, creo que las diferencias entre ambas formulaciones no son relevantes para la discusión presente-como intentaré mostrar en la sección que sigue. Vale la pena notar aquí por otra parte que, si bien, como indica el evaluador, la formulación original de Sidelle (y las de LaPorte y López de Sa tributarias de ella) está presentada en términos que sugieren una interpretación tridimensional, esto es, en términos de objetos que en su totalidad constituyen, aunque por ciertos intervalos limitados de tiempo (y del "espacio modal"), a la entidad inusual, en la formulación anterior, y aparentemente independiente, de Linsky, estas mismas entidades son presentadas en lenguaje que sugiere una lectura tetradimensional de la entidad en cuestión: “[La expresión 'el primer ministro'] puede ser tomada como designando al concepto individual de quien sea corrientemente el primer ministro, o bien a un concepto "individual" verdadero de estadios temporales de distintos individuos en tanto son primer ministro, Joe Clark primero, Pierre Trudeau más tarde, Brian Mulroney aún más tarde" (Linsky 1984, 268-9).

9 Nótese que, en un marco ontológico abundante como el que se está presuponiendo en el contexto de la presente argumentación, la afirmación contenida en la primera oración de esta cita no está suficien- 
Según López de Sa, una situación exactamente análoga a la que acabamos de describir para el caso de algunos términos singulares tiene lugar respecto de ciertas expresiones predicativas, en el sentido de que la aceptación de la existencia de, para volver a nuestro ejemplo, una propiedad como la de ser del color del cielo, tendría que afectar tanto (es decir, tan poco) a nuestra evaluación de un enunciado como (2) como la existencia de El-jefe-de-la-Iglesia-Católica afectaba nuestra evaluación intuitiva de (3). En este sentido, nuestro autor se esfuerza por mostrar que la evaluación intuitiva de (2) como una verdad contingente, que es en su opinión la evaluación natural de dicho enunciado, es tan poco problemática, y tan independiente de presuposiciones metafísicas, como lo es la evaluación correspondiente respecto de (3), ya que ambos casos pueden describirse y explicarse de un modo completamente paralelo. En efecto, podría decirse que, dado que 'ser azul' no parece designar, respecto del mundo actual, a la propiedad de ser del color del cielo, es de esperar que esta propiedad tampoco sea lo designado por 'ser del color del cielo' sino que, por el contrario, habría que suponer que ambas expresiones designan la misma propiedad de ser azul. De este modo, se validaría la premisa (i) de nuestro argumento-y, dada la plausibilidad de sus demás pasos, su conclusión.

La argumentación de López de Sa que venimos de reseñar presenta similitudes notables con algunas consideraciones presentadas con anterioridad por Joseph LaPorte (2000) para defender su concepción de la rigidez frente a una acusación análoga de trivialización; de hecho, parece probable que estas últimas hayan inspirado en buena medida la propuesta de López de Sa. Sin embargo, hay entre sus dos posiciones algunas diferencias significativas; en particular, como ya notamos, LaPorte propone su noción de rigidez para caracterizar a designadores de géneros que son términos singulares corrientes, esto es, nombres o descripciones definidas de los géneros. Esta circunstancia le permitirá en principio aplicar la noción usual de rigidez para términos singulares a estos nuevos casos, que sólo se distinguen de los más corrientes ya discutidos por Kripke en que sus designata son objetos abstractos de un tipo particular - esto es, en este caso, géneros. Como también sugerí más arriba, buena parte del interés que podría despertar la propuesta de LaPorte parecería perderse por esta circunstancia, más en particular por las siguientes razones: en primer lugar, porque no aportaría ninguna novedad respecto del análisis de la noción de rigidez ya realizado por Kripke; en segundo lugar, porque una propuesta de ese tipo resulta excesivamente limitada en su alcance, debido a que, como sugeríamos más arriba, parece suficientemente claro que muchos de los términos de género natural que Kripke considera alcanzados por la tesis de la rigidez son expresiones predicativas; $y$, finalmente, porque tampoco parece poder derivarse a partir de su propuesta una solución a la pregunta, al menos independientemente interesante, de si, y en ese caso en qué sentido, la noción de rigidez podría ser utilizada para caracterizar el comportamiento de expresiones predicativas (ya

temente justificada. En efecto, el carácter contingentemente verdadero de un enunciado como (3) podría ser explicado, tanto por el hecho de que las dos expresiones singulares involucradas designen (una rígida, otra flexiblemente) a Ratzinger, como por el hecho de que ambas expresiones designen a El-jefe-de-la-Iglesia-Católica (aunque en este último caso habría una variación respecto de cuál de las expresiones lo haría rígida y cuál flexiblemente). 
que, al contrario de lo que ocurre con las nominalizaciones analizadas por López de $\mathrm{Sa}$, no hay una conexión regular entre los designadores singulares considerados por LaPorte y las expresiones predicativas correspondientes). Sin embargo, los planteos de ambos autores tienen tantos puntos en común que, como espero hacer ver en lo que sigue, un análisis comparativo de sus propuestas resultará particularmente instructivo.

LaPorte pretende, al igual que López de Sa, argumentar en favor de la existencia de designadores de géneros no rígidos sobre la base de la supuesta verdad contingente de ciertos enunciados de identidad, tales como los siguientes:

(4) La abeja es la especie de insecto típicamente criada para obtener miel.

(5) Soda = la bebida que mi tío pide en las fiestas de Super Bowl.

La argumentación en favor del carácter no rígido de algunas de las expresiones involucradas en estas identidades sigue un patrón previsible a la luz de lo discutido más arriba en relación con la propuesta de López de Sa. Así, por ejemplo, si suponemos que (4) es verdadera, se seguiría que 'la abeja' designa a la misma entidad (al mismo género) que designa 'la especie de insecto típicamente criada para obtener miel'. Pero dado que la especie típicamente criada para obtener miel podría haber sido, por ejemplo, alguna especie de hormiga cuya actividad principal fuera robar miel de las abejas, esta identidad es contingente, con lo que alguno de los dos designadores involucrados, en este caso el que aparece a la derecha del 'es' de identidad, resultaría no rígido.

Todo esto parece perfectamente aceptable y, de hecho, como nuestro autor mismo señala, las consideraciones respecto de 'la abeja' y 'la especie típicamente criada para obtener miel' son completamente paralelas a las que motivan la suposición de que, mientras que 'Héspero' es rígido, 'el objeto celeste más brillante al atardecer' no lo es; como sugeríamos más arriba, más que paralelo el fenómeno es en rigor exactamente el mismo: se trata en ambos casos del contraste entre rigidez y no rigidez para términos singulares, aplicado en un caso a la designación de objetos concretos y en el otro a la de géneros abstractos. Por otro lado, en buena parte por este motivo, no tenemos aquí un tipo de dificultad que encontrábamos en la argumentación de López de Sa: en efecto, en este caso, las intuiciones que se espera que tengamos frente a los ejemplos relevantes no resultan particularmente problemáticas, ya que (4) y (5) parecen efectivamente ser verdaderas de modo contingente.

Además de esta estrategia general, nuestro autor también comparte con López de Sa, por un lado, su preocupación por el problema de la trivialización y, por el otro, el uso de un argumento del tipo que estamos examinando como parte de su respuesta a este problema. Así, al anticiparse a una posible crítica—que, de hecho, no es más que una variante de la objeción de trivialización—señala:

Supóngase, por ejemplo, que además de un género soda, que resulta ser el que mi tío pide, existe el género bebida que mi tío pide en las fiestas de Súper Bowl (llamémoslo 'el género-BMTPFSB') [...] Ahora bien, podría objetarse que 'la bebida que mi tío pide en las fiestas de Súper Bowl' de hecho designa [actually designates] el género BMTPFSB en lugar del género soda. Si esto es así, entonces la expresión 'la bebida que mi tío pide en las fiestas de Súper Bowl' designaría su género, al parecer, rígidamente. Pues, en todo mundo posible, algo es un miembro del género BMTPFSB exactamente cuando es la bebida que mi tío pide en las fiestas de Súper Bowl. De aquí, parecería que 'la bebida que mi tío pide en las fiestas de Súper Bowl' es después de todo un designador rígido, y que no designa de modo no rígido a la soda (LaPorte 2000, 300). 
El problema presentado en este pasaje es, como puede apreciarse, análogo al que preocupaba a López de Sa, a saber: ¿por qué, si se admite la existencia de géneros inusuales, una expresión descriptiva aparentemente hecha a medida para representar uno de dichos géneros designaría algo distinto de ese género? Nuevamente, al igual que ocurría en el caso de López de Sa, la respuesta frente a la objeción consiste en señalar que las expresiones que designan propiedades se comportan, en los aspectos relevantes, de un modo similar a cómo lo hacen las descripciones definidas ordinariases decir, aquellas que designan particulares concretos. Podemos tomar estas consideraciones como expresando un argumento cuya estructuración es paralela a la del que presentaba López de Sa (la única diferencia radica en las clases de expresiones a las que se adscribe comportamientos paralelos):

(i') La influencia que tendría la postulación de una ontología abundante o escasa respecto de la aceptación del carácter rígido o no rígido de ciertos términos es prima facie similar para los casos de los designadores de objetos concretos y de los designadores de propiedades.

(ii') Si esas relaciones entre ontología aceptada y rigidez pueden dar lugar a un problema de trivialización respecto de la rigidez de los designadores de propiedades, también deben dar lugar a un problema similar respecto de los designadores de objetos concretos.

(iii') No hay problema de trivialización para la noción de rigidez aplicada a designadores de objetos concretos.

(iv') No hay problema de trivialización para la noción de rigidez aplicada a designadores de propiedades.

La premisa (i'), nuevamente, es justificada sobre la base de las similitudes relevantes que se dan entre enunciados como (4) y (5), por una parte, y enunciados como (3), por la otra: así como la existencia de El-jefe-de-la-iglesia-católica no impide que 'El jefe de la iglesia católica' sea utilizado para designar de modo no rígido a Ratzinger, la existencia del género BMTPFSB no tiene por qué impedir que 'la bebida que mi tío pide en las fiestas de Súper Bowl' designe al género soda de modo no rígido, tal como sugiere la evaluación intuitiva que hacemos de ese enunciado. De este modo, entonces, LaPorte pretende mostrar que la aceptación de una ontología abundante respecto de las propiedades no tiene por qué generar un problema de trivialización para su concepción de la rigidez para designadores de géneros.

\section{Evaluación de los argumentos considerados y sus presupuestos}

Hasta aquí nos hemos dedicado a presentar, por una parte, un cierto tipo de argumentación que involucra la aceptación de ciertas tesis respecto de las conexiones entre ontología y rigidez y, por otra parte, el contexto argumentativo más general en que cabe inscribir esos pasos. Corresponde ahora evaluar estas argumentaciones y sus presupuestos. Digamos en primer lugar, respecto de la propuesta de López de Sa, que su defensa de (i) no parece particularmente convincente debido a que, contrariamente a lo que él supone, los dos tipos de expresiones cuyo comportamiento semántico allí se asimila funcionan de modos tan diferentes que la analogía no puede sostenerse. En 
efecto, nótese que, mientras que las nominalizaciones de predicados del tipo de 'ser del color del cielo', parecen ser aptas, desde un punto de vista formal, para designar las entidades abundantes correspondientes (en este caso, la propiedad ser del color del cielo), no parece que ocurra lo mismo con las descripciones definidas, tales como 'el papa': como intentaré mostrar en lo que sigue, parece que una expresión de este último tipo no podría, en verdad, designar a una entidad tal como El-jefe-de-la-Iglesia-Católica-y ello por razones puramente semánticas, independientes de las consideraciones metafísicas mencionadas por nuestro autor.

¿Cuáles serían las razones para suponer que esto es así? Permítaseme considerar en primer lugar, para facilitar la exposición, un ejemplo ligeramente distinto del que venimos analizando (hasta donde puedo ver, nada sustancial dependerá de esta elección). Considérese la descripción 'el ser humano que se encuentra a mayor altitud sobre el nivel del mar'. Pareciera que, en relación con ella, podríamos presentar a la personade-cargo El-ser-humano-que-se-encuentra-a-mayor-altitud-sobre-el-nivel-del-mar que, según López de Sa, podría ser aquello designado rígidamente por dicha descripción. Pero, ¿puede efectivamente esa descripción designar a esa persona-de-cargo? En mi opinión, es claro que no puede hacerlo. Nótese en primer lugar que se sigue del significado mismo de una descripción como 'el ser humano que se encuentra a mayor altitud sobre el nivel del mar' que aquello que vaya a ser designado por semejante expresión tiene que satisfacer la condición descriptiva incluida en ella. En particular, en este caso, una condición necesaria que tiene que cumplir todo aquello que vaya a ser designado por esta descripción (dejando de lado casos del tipo que ha discutido Donnellan) ${ }^{10}$ es la de ser un ser humano. Pero, ¿es El-ser-humano-que-se-encuentra-amayor-altitud-por-sobre-el-nivel-del-mar un ser humano? Me parece suficientemente claro que no lo es, con lo que semejante entidad no podría ser, pace López de Sa y, junto con él, LaPorte, Linsky y probablemente también Sidelle, aquello designado por nuestra descripción. Ciertamente, ciertos estadios de dicha entidad coinciden con ciertos estadios de seres humanos (en virtud de que tales estadios constituyen parcialmente aquella entidad), pero esta entidad inusual no es, ella misma, un ser humano. ${ }^{11} \mathrm{Si}$

${ }^{10}$ Los "casos tipo Donnellan" son casos de usos de descripciones en los que el hablante logra designar a un individuo particular aun cuando éste no cumpla con todas las condiciones identificadoras especificadas en la descripción (cf. Donnellan 1966). Se suele suponer, de todos modos, que estos son usos no estándar de las descripciones, en los que la designación tiene lugar a pesar de que la condición descriptiva no es satisfecha.

11 Corresponde intentar despejar aquí una duda planteada por un evaluador anónimo, a saber, si esta conclusión no podrá sostenerse sólo debido a la aceptación (implícita) de una interpretación específica, a saber, una tetradimensional, de la entidad inusual. En efecto, el lenguaje tridimensional utilizado por Sidelle sugiere que una persona usual, la totalidad de ella en cada momento, constituye, a lo largo de un cierto período, a la entidad inusual en cuestión. Esto puede hacer creer, y probablemente así lo creyó Sidelle, que 'persona' podría aplicarse a cada uno de tales estadios. Pero, en primer lugar, parece que, si 'persona' conlleva (como parece razonable suponer) un criterio de individuación definido, 'persona' sólo puede aplicarse en un cierto momento a la persona usual que constituye, durante un intervalo que incluye a ese momento, a una entidad como El-jefe-de-la-Iglesia-Católica, pero no a la entidad inusual misma, ya que, como el mismo Sidelle acepta, estos dos objetos difieren en sus condiciones de individuación (1992, 417, 420; la situación sería entonces semejante a la bien conocida del trozo de arcilla y la estatua: si bien ambas coinciden durante cierto intervalo temporal, 'sobrevive a un 
bien esto puede verse de modo más claro en este ejemplo, consideraciones similares se aplican respecto de entidades como El-jefe-de-la-Iglesia-Católica y expresiones como 'el papa', suponiendo que esta última tiene el valor de una descripción como 'la persona que es jefe de la Iglesia Católica’.

Quizás ayude a comprender con mayor claridad lo que venimos de decir la consideración de los distintos valores de verdad que cabría atribuir a ciertos enunciados, según se suponga que descripciones tales como 'el papa' designen a uno u otro tipo de entidad. En este sentido, creo que López de Sa se equivoca al decir lo que sigue:

[...] la suposición de que 'el papa' contribuye de manera uniforme a las condiciones de verdad de oraciones simples como 'El papa es alemán' el objeto [abundante] El-jefe-de-la-Iglesia-Católica parece dar los mismos resultados respecto de la evaluación actual y contrafáctica de dichas oraciones que nuestra suposición original que involucraba los distintos objetos [escasos] Ratzinger y Aznar (2008, 6a).

Según creo, que las cosas resulten o no tal como lo supone aquí López de Sa dependerá del tipo de propiedad que se atribuya, en cada caso, a los objetos designados por las descripciones en los enunciados simples relevantes, pero, en todo caso, parece que lo que se dice en esta cita es, de un modo general, falso. La situación puede no ser del todo clara respecto de casos como el del ejemplo que él presenta aquí ('El papa es alemán'), pero hay otros casos cuya evaluación resulta mucho menos dudosa. Por ejemplo, si bien podemos suponer que un enunciado mediante el cual se adscribe al objeto designado por 'el papa' una propiedad claramente ocurrente, tal como la de ser gordo, habrá de ser evaluada como teniendo un mismo valor de verdad, tanto si consideramos que la descripción refiere de manera rígida a El-jefe-de-la-Iglesia-Católica como si consideramos que refiere de manera no rígida a los distintos individuos que han sido (o podrían haber sido) papas, esto no es así en lo que respecta a todas las propiedades que podríamos adscribir a aquello designado por 'el papa'. En efecto, la situación parece ser distinta cuando se trata de adscribir propiedades relacionadas, o bien con la carrera (la historia), o bien con las condiciones de individuación, de los objetos relevantes, respecto de las cuales la suposición de que un término designa a uno u otro objeto puede dar lugar a distintas asignaciones de valores de verdad a los enunciados en los que se formulan tales adscripciones. Tomemos, por ejemplo, la propiedad de ser humano. Supongamos que adscribimos esta propiedad en un enunciado como (6):

reamasado' se aplica con verdad sólo al trozo de arcilla, y no a la estatua que aquella constituye; de una manera similar, '... es una persona', en tanto que se aplique con verdad a nuestra entidad inusual, tiene que entenderse como involucrando un 'es' de constitución, no de predicación (esto es, como teniendo el valor de 'está constituido por una persona', no el de 'es, ella misma, una persona'); para una discusión clásica de estos temas cf. Wiggins 2001, cap. 1); si, por otra parte, se supone que 'persona' no conlleva condiciones de individuación definidas, de modo que fuera equivalente a (algo así como) 'entidad de carácter personal', debería poder aplicarse a cualquier entidad que coincidiera con algún estadio de una persona; si esto fuera así, típicamente las descripciones que incluyeran tal noción en sus condiciones descriptivas serían impropias (habría siempre demasiados candidatos entre los que escoger; en nuestro ejemplo, por caso, tanto la persona usual como la persona-de-cargo coincidentes), lo que no concuerda con nuestra apreciación corriente de muchas de esas descripciones. Si lo que vengo de decir es correcto, entonces, la argumentación presentada en el cuerpo del artículo se sostiene con independencia del modo en que se suponga que tales objetos persisten. 
(6) El papa es un ser humano.

Como sugeríamos más arriba, pareciera que este enunciado debería ser evaluado como verdadero si suponemos que 'el papa' designa, con respecto al mundo actual, a Joseph Ratzinger (en cuyo caso la expresión sería no rígida), pero que deberíamos evaluar ese mismo enunciado como falso si supusiéramos que dicha expresión designa a la entidad inusual El-jefe-de-la-Iglesia-Católica. Algo similar ocurre con un ejemplo como (7):

(7) El papa nació en Alemania.

Si entendemos 'el papa' como un designador no rígido e inconstante de las distintas personas usuales que han sido (o podrían haber sido) papas en las distintas circunstancias (tanto modales como temporales) relevantes para la evaluación de un enunciado, de acuerdo con lo cual dicha descripción designaría, respecto de finales de 2012 y el mundo actual, a Joseph Ratzinger, (7) resultará ser verdadera. Pero, por el contrario, si suponemos que 'el papa' designa rígida y constantemente a aquella otra entidad inusual, a El-jefe-de-la-Iglesia-Católica, dicho enunciado presumiblemente no es verdadero, ya que la propiedad de haber nacido en algún lugar determinado, según parece, ni siquiera estaría bien definida para un objeto como El-jefe-de-la-Iglesia-Católica (dejo abierto si en este caso el enunciado sería falso o simplemente carente de valor de verdad)..$^{12}$

En otros casos, finalmente, en los que las descripciones interactúan con operadores temporales y modales, las diferencias entre ambos modos de entender la referencia de las descripciones se manifiestan, no directamente mediante valores de verdad contrastantes, sino mediante la existencia (o inexistencia) de ciertas ambigüedades de alcance (como es de esperar cuando analizamos pares de expresiones que se distinguen por su rigidez). Considérese el siguiente ejemplo:

(8) El papa se encontraba en Alemania al estallar la segunda guerra mundial.

Este enunciado parece ser ambiguo, según cuál sea el alcance que se dé al operador temporal 'al estallar la segunda guerra mundial'. En efecto, si tomamos a esta expresión con un alcance amplio, de modo que modifique la totalidad del contenido proposicional expresado por el resto del enunciado, la adopción de una u otra hipótesis respecto del significado de la descripción no daría lugar a diferencias en los valores de verdad de los enunciados resultantes; presumiblemente, (8) sería falsa en cualquiera de esas hipótesis (debido a que, al momento de estallar la guerra, la persona que era papa en ese momento no estaba, según podemos conjeturar, en Alemania). Pero si se considera que la descripción está por fuera del alcance del operador (es decir, si se hace de ella una lectura de re), entonces la suposición de que ella tiene uno u otro tipo de comportamiento tendrá consecuencias respecto del valor de verdad que le asignemos al

${ }^{12}$ En general, parece que siempre que se trate de propiedades que el papa podría tener durante algún intervalo del curso efectivo de su papado, la interpretación inusual sería aceptable. Los ejemplos en que esto no ocurriría serían casos donde inequívocamente se adscribe una propiedad que el papa no podría tener en tanto papa (por ejemplo, expresiones de la forma 'el papa fue / era F antes de ser nombrado papa'). 
enunciado: en efecto, mientras que éste será presumiblemente verdadero si se considera que la descripción refiere a la entidad usual Ratzinger, será por el contrario falso si se considera que aquella designa a la entidad inusual propuesta, ya que quien era papa en ese momento, que constituye un estadio temporal de dicha entidad, no estaba, según supusimos más arriba, en Alemania en ese momento (suponiendo, claro está, que esta conjetura acerca del mundo actual sea correcta). Es decir que, mientras que la suposición de que la descripción designa a la entidad usual permite capturar la lectura intuitivamente verdadera de (8), tal resultado no es posible si se sostiene que la descripción designa rígidamente a la entidad inusual correspondiente.

Algo similar ocurre en casos que involucran propiedades modales. ${ }^{13}$ Consideremos este ejemplo:

(9) El papa podría no haber sido un eclesiástico.

Parece claro que hay una lectura de (9) según la cual ella resulta verdadera, pero no podría haberla si 'el papa' designase a El-jefe-de-la-Iglesia-Católica, ya que no habría circunstancia en que no fuera cierto de esa entidad que pertenece a la iglesia. La lectura intuitivamente verdadera de (9) parece sugerir, entonces, que 'el papa' no designa a tal entidad inusual. ${ }^{14}$

Todo lo que venimos de decir respecto de las descripciones contrasta con lo que ocurre en el caso de las nominalizaciones canónicas, en cuyo caso nada impediría, según parece, que las entidades "inusuales" resulten ser designadas por ellas—y, más aún, tales propiedades inusuales parecen ser justamente aquello que tales nominalizaciones designarían del modo más natural. En efecto, es claro que, al menos en algunas

13 Puede dudarse, como lo ha hecho un evaluador anónimo, de la pertinencia de apelar a ejemplos que involucran predicaciones modales en la situación dialéctica presente, ya que López de Sa explícitamente restringe su tratamiento a las que denomina "oraciones simples", esto es, oraciones sin conectivas lógicas ni operadores intensionales. Hay varias observaciones que pueden hacerse a este respecto: en primer lugar, que no todos los ejemplos que hemos presentado involucran nociones modales (por ejemplo, los referidos a la historia de la entidad en cuestión no lo hacen), de modo que las dificultades para su posición aún persistirían; en segundo lugar, que estas restricciones tenidas en cuenta por López de Sa se originan, no tanto en problemas ligados al tipo de evidencia que sería admisible para resolver la cuestión de la rigidez, sino en un intento de evitar ciertas dificultades al caracterizar a los valores semánticos de las descripciones como los particulares denotados por ellas; finalmente, que no se ve por qué, en general, la discusión acerca de la trivialización debería adoptar esta restricción; de hecho, algunos autores, como Martí y Martínez (2010) discuten explícitamente casos con operadores intensionales como los únicos que permitirían solucionar dicho problema.

${ }^{14}$ Como es de esperar, una crítica de este tipo no es del todo concluyente; como ya hacía notar de modo general David Lewis (1983, x), salvo raras excepciones "la teoría sobrevive a su refutación-a un cierto precio". López de Sa podría apelar, para defender su posición, a una teoría no standard de la predicación, según la cual las propiedades históricas, modales y clasificatorias (sortals) sean verdaderas de objetos inusuales según cuál sea la historia, actual y contrafáctica, y el género, de los distintos objetos usuales cuyas partes modales y temporales constituyen al objeto inusual en cada una de las circunstancias de evaluación relevantes. Esto implicaría, entonces, que ciertos predicados, como por ejemplo, 'haber nacido en Alemania' deberían entenderse de modos distintos si se concatenan con designadores de entidades usuales o de entidades inusuales. Si bien posiciones de este tipo han sido defendidas en la literatura (por ejemplo, Harold Noonan ha defendido que ciertos predicados son "abelardianos", es decir, que cambian de significación según cuál sea el objeto al que se aplican; cf. Noonan 1991), creo que podrá concedérseme que éste es un precio demasiado alto. 
ocasiones, 'ser del color del cielo' podría designar la propiedad de ser del color del cielo, y que, en todo caso, no hay nada que impida que dicha expresión designe tal propiedad de manera sistemática, si se acepta que dicha propiedad existe (al contrario de lo que ocurre en los casos de descripciones singulares que venimos de ver); y, dada la conexión sistemática entre nominalizaciones canónicas y predicados, ligada en buena medida a que la nominalización no pierde completamente su carácter verbal y, en consecuencia, predicativo, es igualmente razonable suponer que los predicados correspondientes se comportan también de modo rígido (adscribiendo la misma propiedad respecto de todos los mundos posibles). En todo caso, lo que estos ejemplos parecen mostrar de modo suficientemente claro es que los comportamientos relevantes de las descripciones definidas, por un lado, y los de las nominalizaciones y expresiones predicativas consideradas, por el otro, no son de ningún modo paralelos: en efecto, mientras que no parece haber ninguna razón basada en su comportamiento semántico que impida suponer que las expresiones predicativas supuestamente "descriptivas" son sistemáticamente rígidas del modo requerido, sí parece haber tales motivos en el caso de las descripciones definidas; en efecto la suposición de que tales descripciones fueran rígidas debido a que designarían cierto tipo de entidad inusual no puede hacerse concordar de un modo natural, ni con consideraciones básicas acerca de su funcionamiento semántico, ni con la evaluación intuitiva que, según venimos de ver, hacemos respecto de predicaciones simples tales como 'El papa es un ser humano' o 'El papa nació en Alemania'; mientras que, justamente, lo contrario tiene lugar para el caso de los términos generales.

¿Cuánto de lo que venimos de decir se aplica también a lo sostenido por LaPorte? Notemos en primer lugar que la tesis de paralelismo (i') parece perfectamente aceptable en este caso, ya que, como notamos en la sección anterior, las dos clases de términos comparadas allí, distinguidas sólo por diferencias en sus objetos, están constituidas en ambos casos por expresiones de un tipo formalmente similar-esto es, están constituidas en ambos casos por expresiones que cabe considerar como términos singulares (ya sean nombres o descripciones). Esto implica que las expresiones contenidas en las dos clases comparadas allí se comportan más bien como lo hace el ejemplo 'el papa' considerado en la discusión anterior, en lugar de como lo hace el ejemplo 'ser del color del cielo'. De esto se sigue que este tipo de expresiones no podría en verdad designar a las entidades inusuales que LaPorte supone que sí podrían designarentidades como el género BMTPFSB o el objeto El-jefe-de-la-Iglesia-Católica. Por cierto, si bien esto implica que él no debería haber aceptado como plausible una objeción que descansaba justamente en esta suposición dudosa, que por lo tanto habría correspondido rechazar por los motivos que venimos de señalar, el resultado no es por sí mismo particularmente devastador para su propuesta: al contrario, al dar motivos adicionales para rechazar la objeción, las consideraciones previas más bien refuerzan antes que comprometen su posición—aunque, por cierto, su propuesta puede ser criticada por otros motivos: en particular, como notábamos más arriba, por los problemas de generalidad derivados de que quedarían fuera del alcance de su explicación muchos términos de género natural que sólo cabría comprender como predicativos. Pero, en todo caso, el hecho mismo de que LaPorte haya aceptado la objeción como plausible 
nos permite ver que este autor comparte con López de Sa una concepción respecto de cuál sería el rango de referentes aceptables para ciertas descripciones que, según intentamos mostrar, es errónea-justamente, se trata de la concepción criticada más arriba según la cual se puede incluir dentro de ese rango entidades inusuales como las que estuvimos considerando.

Viene al caso preguntarse, finalmente, acerca de los motivos que podrían estar a la base de la aceptación de un presupuesto semejante. Puede notarse en una primera instancia, en este sentido, cierta tendencia a asimilar el valor semántico de una frase referencial, tal como 'el color del cielo', por una parte, con el que se puede asignar, por la otra, a un término general, tal como 'del color del cielo'-tendencia que parece estar también a la base de la suposición de López de Sa de que sus nominalizaciones canónicas podrían ser no rígidas y, correlativamente, de que enunciados como (1) y (2) son, intuitivamente, verdades contingentes. Si nos preguntamos a su vez ahora por los motivos de esta asimilación, quizás puedan mencionarse, entre otros que se me escapan, los dos siguientes: en primer lugar, que en inglés, lengua que ha sido el vehículo de la casi totalidad de la discusión sobre el tema, las expresiones equivalentes a esas dos castellanas son indistinguibles (en efecto, se puede usar 'the colour of the sky' tanto para referirse al color del cielo en cada circunstancia como para decir de algo que es del color del cielo, cuando aquella expresión aparece en el contexto de un predicado como ' $\ldots$ is the colour of the sky'); ${ }^{15}$ por otra parte, parece que también podría haber a veces cierta intromisión, a la hora de interpretar intuitivamente estas expresiones predicativas, de ciertos presupuestos "escasos" en relación con el tipo de entidad en cuestión. Así, por ejemplo, Armstrong, que considera explícitamente una expresión como 'del color que Ángela prefiere’ (of the colour Angela prefers), sostiene que dicha expresión predicativa puede ponerse en correlación con universales distintos en diversas circunstancias (1978, Vol. II, 57); pero, en todo caso, debería ser suficientemente claro que, como sea que se explique el fenómeno en cuestión-ya sea en términos de la "realización" de una propiedad de nivel superior por parte de otras más básicas, ya en términos de distintos universales que contribuirían a hacer verdaderos los enunciados en que aparecen esos predicados-lo que tiene lugar en tales casos es un fenómeno completamente distinto del mecanismo semántico mediante el cual un objeto es designado por una descripción, como cabría esperar que ocurriera si fuera a justificarse por motivos semánticos la asimilación que estamos discutiendo. En todo caso, sean estos u otros los motivos que la explican, es claro que la asimilación en cuestión parece infundada.

Querría finalizar mi discusión de estas propuestas sugiriendo que la asimilación de estos dos tipos de expresiones que venimos de señalar, así como la correlativa desatención de ciertas diferencias formales entre las expresiones involucradas, permitirían

15 Lo dicho aquí no implica negar que también pueda haber usos predicativos de frases del tipo de 'el color del cielo' (esto es, descripciones definidas), aun cuando no se las asimile a frases tales como 'del color del cielo'. Así, por ejemplo, en 'Napoleón no fue el mejor militar francés' no se indica la no identidad entre Napoleón y aquello designado por 'el mejor militar francés', sino que se dice de Napoleón que no tiene la propiedad de ser el mejor militar francés (para una discusión de los usos predicativos de las descripciones cf. Fara 2001). 
quizás explicar la suposición, central para los autores que estamos examinando, de que las expresiones analizadas en sus propuestas pueden tener propiedades que, bien miradas, son en última instancia incompatibles: a saber, por un lado, la de ser no rígidas y, por otro, la de designar las propiedades que se atribuyen mediante ellas de modo que, por decirlo así, se refleje en su propia estructura la de la propiedad en cuestión. Así, mientras que las descripciones consideradas por LaPorte manifiestan un tipo de complejidad que les permite designar (posiblemente distintas) propiedades en virtud de cumplir estas con ciertas condiciones (típicamente contingentes), como consecuencia de lo cual esas descripciones pueden (y típicamente son) no rígidas, esa característica suya les impide expresar aquello que se adscribe a un objeto cuando se le adscribe la propiedad en cuestión; mientras que justamente lo opuesto ocurre con las nominalizaciones analizadas por López de Sa: su derivación regular a partir de predicados, y la consiguiente conservación de cierto aspecto adscriptivo (i.e., verbal), les permite representar las propiedades que designan (reproduciendo en ellas mismas, por así decir, la estructura de esas propiedades) de un modo en que no lo puede hacer una descripción del tipo mencionado más arriba, aun cuando esta misma capacidad parece ser, por otra parte, lo que les impide relacionarse semánticamente con distintas propiedades (esto es, lo que les impide ser no rígidas). ${ }^{16}$ Podemos presentar esta diferencia de un modo alternativo, si consideramos a qué se aplica el contenido descriptivo presente en las distintas expresiones relevantes. Así, mientras que las descripciones de propiedades como las que tiene en cuenta LaPorte son tales que ese contenido descriptivo es verdadero de la propiedad misma de objetos de primer orden así seleccionada, y no de los objetos que la instancian, en el caso de las nominalizaciones consideradas por López de Sa su contenido descriptivo sí puede utilizarse para describir, por el contrario, a los objetos de primer orden a los que se aplican los predicados a partir de los cuales dichas nominalizaciones fueron formadas. Esto permite ver también por qué, en un nivel más profundo, sólo las nominalizaciones del tipo que analiza López de Sa podrían ser relevantes para la cuestión de definir una noción de rigidez para expresiones predicativas, ya que sólo ellas pueden considerarse como (adecuadamente relacionadas con) expresiones genuinamente predicativas, en el sentido de que se trate de expresiones que permitan adscribir propiedades, y no meramente designarlas.

\section{Conclusiones}

Si nuestra argumentación precedente está bien encaminada, los argumentos de LaPorte y López de Sa que venimos de examinar deben ser rechazados debido a que se basan en presupuestos erróneos respecto de qué entidades podrían ser, prima facie, referentes aceptables de las descripciones definidas. Por otra parte, vale la pena señalar que, si bien esos presupuestos ( $\mathrm{y}$ los argumentos basados en ellos) cumplen un papel

${ }^{16} \mathrm{El}$ contraste que queremos expresar de este modo tiene ciertas similitudes con el que Armstrong ofrece entre los que denomina "predicados externos" y "predicados analizantes": mientras los últimos identifican una propiedad diciendo algo "acerca de la naturaleza interna del universal en cuestión" (Armstrong 1978, Vol. II, 57), los primeros lo hacen "de modo indirecto o externo, a través de sus propiedades (de segundo orden)" (esta última frase proviene de la discusión de una distinción conexa en Linsky 1984, 268). 
relativamente marginal dentro de la estrategia argumental desplegada por cada uno de estos autores, su análisis permite arrojar luz, como creo haber mostrado hacia el final de la sección precedente, sobre las razones por las cuales sus propuestas resultan en última instancia insatisfactorias a la hora de formular de manera precisa una noción de rigidez aplicable a expresiones predicativas.

\section{REFERENCLAS}

Armstrong, David. 1978. Universals and Scientific Realism. Cambridge: Cambridge University Press. Donnellan, Keith. 1966. Reference and Definite Descriptions. The Philosophical Review 75: 281-304.

Fara, Delia Graff. 2001. Descriptions as Predicates. Philosophical Studies 102: 1-42 (publicado originalmente con el nombre 'Delia Graff').

Gómez-Torrente, Mario. 2006. Rigidity and Essentiality. Mind 115: 227-259.

Kripke, Saul. 1980. Naming and Necessity. Cambridge, MA: Harvard University Press.

LaPorte, Joseph. 2000. Rigidity and Kind. Philosophical Studies 97: 293-316.

—. 2006. Rigid Designators for Properties. Philosophical Studies 130: 321-336.

Lewis, David. 1983. Philosophical Papers I. Oxford: Oxford University Press.

-. 1986. On the Plurality of Worlds. Oxford: Blackwell.

Linsky, Bernard. 1984. General Terms as Designators. Pacific Philosophical Quarterly 65: 259-276.

—. 2006. General Terms as Rigid Designators. Philosophical Studies 128: 655-667.

López de Sa, Dan. 2008. Rigidity for Predicates and the Trivialization Problem. Philosopher's Imprint 8 (1): $1-13$.

Martí, Genoveva, y Martínez, José. 2010. General Terms as Designators: A Defence of The View. En The Semantics and Metaphysics of Natural Kinds, editado por Helen Beebee y Nigel Sabbarton-Leary, 46-63. Londres: Routledge.

—. 2011. General Terms, Rigidity and the Trivialization Problem. Synthese 181: 277-293.

Noonan, Harold. 1991. Indeterminate Identity, Contingent Identity and Abelardian Predicates. The Philosophical Quarterly 41: 183-193.

Sidelle, Alan. 1992. Rigidity, Ontology and Semantic Structure. The Journal of Pbilosophy 89: 410-430.

Wiggins, David. 2001. Sameness and Substance Renewed. Cambridge: Cambridge University Press.

Ezequiel Zerbudis es Licenciado y Doctor en Filosofía por la Universidad de Buenos Aires, docente en dicha institución en las áreas de Filosofía del Lenguaje e Historia de la Filosofía Moderna, y docente en la Universidad Nacional del Litoral, Argentina, en el área de Filosofía del Lenguaje. Ha presentado ponencias y publicado trabajos sobre temas en dichas áreas, y en metafísica contemporánea, en revistas tales como Análisis Filosófico, Archiv für Geschichte der Philosophie, Revista Latinoamericana de Filosofía y Teorema.

Dirección: Departamento de Filosofía, Universidad de Buenos Aires, Puán 480, 1406 Ciudad de Buenos Aires, Argentina. E-mail: ezerbu@yahoo.com 